\title{
The role of quercetin in membrane stability
}

Dear Editor,

a recent paper by Margina et al. has shown that quercetin and epigallocatechin gallate induced a dose-dependent increase of membrane anisotropy and polarization in U937 monocytes and Jurkat T lymphoblasts, suggesting that anisotropy increase was correlated with the reduction of lipid peroxidation (Margina et al. 2012). This property has been reported also for erythrocytes.

In mice, the treatment with the bioflavonoids quercetin and diquertin on the physical work capacity, oxidant and antioxidant status of organism, and hemorheological parameters and deformability of erythrocytes have been studied under conditions of chronic physical overstrain provoked by sequential extraordinary running load. Both bioflavonoids significantly increased the physical work capacity of animals and prevent from the development of physical overstrain syndrome (Tipikin et al. 2011). Phenolic extracts from two monofloral Cuban honeys, rich in lipophilic compounds, were shown to possess the ability to inhibit 2,20-azobis(2-methylpropionamidine) dihydrochloride $(\mathrm{AAPH})$-induced oxidative damage (hemolysis, lipid peroxidation and cytosolic depletion of reduced glutathione and decrease of superoxide dismutase activity) in erythrocytes (Alvarez-Suarez et al. 2012). Polyphenolic components showed an important total antioxidant capacity measured by trolox-equivalent antioxidant capacity assay (TEAC) and oxygen radical absorbance capacity assay (ORAC) assays, as well as a relevant radical scavenging activity performed by electron paramagnetic resonance (EPR) spectroscopy. In this study, about a dozen of phenolic compounds were identified using HPLC-LC/MS with quercetin as the most abundant flavonoid (Alvarez-Suarez et al. 2012). A possible target in this mechanism might be phosphatidylinositol-3-kinase (PI3K). Some recently reported evidence has shown that 4hydroxy-2-nonenal (4-HNE) is a major lipid peroxidation product in the retina and the retinal pigment epithelium. When cultured ARPE-19 cells were treated with different concentrations of 4-HNE and a PI3K inhibitor, LY294002, the transcriptional activity of NF-E2-related factor 2 (Nrf2) and GSH increased in a dose-dependent manner (Chen et al. 2009). The modulatory subunit of GCL was up-regulated by 4 -HNE, while antioxidant responses were largely abolished by pretreatment with LY294002; furthermore, the modification of Hsc70 by 4 -HNE was increased when PI3K was inhibited (Chen et al. 2009). LY294002 shares many hallmarks with quercetin (Walker et al. 2000), thus rendering this speculative suggestion a possible next issue for research. The activity of quercetin on PI3K may be ruled by hormetic mechanisms and biphasic action, as previously described (Chirumbolo et al. 2010). However, the counteracting action of quercetin on lipid peroxidation is shared by other phenolics, such as catechins (Kalender et al. 2012). In a more general way, lipid peroxidation is the process of oxidative degradation of polyunsaturated fatty acids and its occurrence in biological membranes causes impaired membrane fluidity and inactivation of a several membrane-bound enzymes. Malondialdehyde (MDA) is one of the major oxidation products of peroxidized polyunsaturated fatty acids, and thus increased MDA content might be considered an important indicator of lipid peroxidation (Demir et al. 2011). The effort to comprehend quercetin targets within intracellular signaling represents a main task for researchers who are trying to elucidate the anti-peroxidative effect of the flavonol. Heme oxygenase-1 (HO-1) catalyzes the rate limiting reaction of heme metabolism and plays critical roles in resistance to oxidative stress and other cellular functions. The enzyme HO-1 is involved also in ROS-mediated peroxidation. HO-1 is induced in response to various stresses but the signaling pathways involved remain incompletely elucidated. The HO1 induction is decreased by phosphatidylinositol 3-kinase (PI3K) inhibitors LY294002 and wortmannin (Zhang and Forman 2008). If the anti-peroxidative effect of quercetin is attributed to the inhibition of PI3K, the anti-oxidant action should be related to Nrf2 and GSH. The hypothesis is that PKC $\delta$ appears to regulate HO-1 induction via modulating Nrf2 nuclear translocation, while PI3K may work through targeting on downstream signaling molecules other than Nrf2 (Zhang and Forman 2008). This suggestion has been reported also for genistein.

This flavonoid alleviated the increase of ROS and nitrotyrosine production induced by $\beta$-amyloid peptide 25-35 (A $325-35)$, and maintained bEND.3 cell redox state by increasing GSH level and GSH/GSSG. In addition, the flavonoid could reverse the down-regulation of total protein and mRNA expression of Nrf2, nuclear Nrf2, $\gamma$-glutamylcysteine synthetase ( $\gamma$-GCS), phosphatidylinositol 3-kinase (PI3K) induced by A $325-35$; while PI3K inhibitor LY294002 could attenuate the activation effects of genistein on Nrf2, especially on nuclear translocation. The potential mechanisms have been associated with the activation of Nrf2 signaling pathway by modulating PI3K activity (Xi et al. 2012). The anti-oxidant effect of quercetin and its ability to reduce lipid 
peroxidation may be considered also a possible cause in the eradication of microbial infection. For example, oral quercetin administration in guinea pigs decreases $H$. pylori infection in the gastric mucosa and reduces both the inflammatory response and lipid peroxidation. In the antrum of infected quercetin-treated animals, a significant diminution of neutrophil leukocyte infiltration was observed compared with the infected non quercetin-treated animals; furthermore, the lipid hydroperoxide concentration in the antrum was significantly decreased in infected animals treated with quercetin, whereas in the corpus no significant differences were observed (Gonzalez-Segovia et al. 2008). This aspect is very intriguing and encourages further research on flavonoids as anti-microbial substances. In the next future, biochemical and genetic studies on cellular and animal models on the mechanism(s) of action of phytochemicals provide a functional explanation of how and why a diet rich in fruits and vegetables is considered healthy. It is not unusual to find molecules that protect against diseases, which greatly differ from a physio-pathological point of view, such as oxidative stress-mediated disorders. Quercetin falls into this category and possesses a broad range of biological properties. Uptake, metabolism and circulating concentrations of quercetin and its metabolites suggest that a regular diet provides amounts of quercetin $(<1 \mu \mathrm{M})$ not compatible with its chemopreventive and/or cardioprotective effects. However, it appears relatively easy to increase total quercetin concentrations in plasma $(>10$ $\mu \mathrm{M})$ by supplementation with quercetin-enriched foods or supplements. Multiple lines of experimental evidence suggest a positive association between quercetin intake and improved outcomes of inflammatory cardiovascular risk. Quercetin can be considered the prototype of a naturally-occurring chemopreventive agent, especially for endothelium, because of its key roles in eliciting an anti-oxidative response, although several critical points must be taken into account when considering the potential therapeutic use of this molecule. The design of specific clinical trials is extremely warranted to depict possible applications of quercetin in oxidative stress and lipid peroxidation.

Salvatore Chirumbolo

Department of Medicine, University of Verona, LURM-Policlinico GB Rossi, Piazzale AL Scuro 10, 37134 Verona, Italy

E-mail: salvatore.chirumbolo@univr.it

\section{References}

Alvarez-Suarez J. M., Giampieri F., González-Paramás A. M., Damiani E., Astolfi P., Martinez-Sanchez G., Bompadre S., Quiles J.
L., Santos-Buelga C., Battino M. (2012): Phenolics from monofloral honeys protect human erythrocyte membranes against oxidative damage. Food Chem. Toxicol. 50, 1508-1516 http://dx.doi.org/10.1016/j.fct.2012.01.042

Chen J., Wang L., Chen Y., Sternberg P., Cai J. (2009): Phosphatidylinositol 3 kinase pathway and 4-hydroxy-2-nonenal-induced oxidative injury in the RPE. Invest. Ophthalmol. Vis. Sci. 50, 936-942 http://dx.doi.org/10.1167/iovs.08-2439

Chirumbolo S., Marzotto M., Conforti A., Vella A., Ortolani R., Bellavite P. (2010): Bimodal action of the flavonoid quercetin on basophil function: an investigation of the putative biochemical targets. Clin. Mol. Allergy 8, 13-24

http://dx.doi.org/10.1186/1476-7961-8-13

Demir F., Uzun F. G., Durak D., Kalender Y. (2011): Subacute chlorpyrifos-induced oxidative stress in rat erythrocytes and the protective effects of catechin and quercetin. Pestic. Biochem. Physiol. 99, 77-81 http://dx.doi.org/10.1016/j.pestbp.2010.11.002

González-Segovia R., Quintanar J. L., Salinas E., Ceballos-Salazar R., Aviles-Jiménez F., Torres-López J. (2008): Effect of the flavonoid quercetin on inflammation and lipid peroxidation induced by Helicobacter pylori in gastric mucosa of guinea pig. J. Gastroenterol. 43, 441-447 http://dx.doi.org/10.1007/s00535-008-2184-7

Kalender Y., Kaya S., Durak D., Uzun F. G., Demir F. (2012): Protective effects of catechin and quercetin on antioxidant status, lipid peroxidation and testis-histoarchitecture induced by chlorpyrifos in male rats. Environ. Toxicol. Pharmacol. 33, 141-148 http://dx.doi.org/10.1016/j.etap.2011.12.008

Margina D., Ilie M., Manda G., Neagoe I., Mocanu M., Ionescu D., Gradinaru D., Ganea C. (2012): Quercetin and epigallocatechin gallate effects on the cell membranes biophysical properties correlate with their antioxidant potential. Gen. Physiol. Biophys. 31, $47-55$ http://dx.doi.org/10.4149/gpb_2012_005

Tipikin I. S., Rozhkova E. A., Seĭfulla R. D., Ordzhonikidze Z. G., Paniushkin V. V., Kravtsov A. A. (2011): Flavonoid correction of functional elasticity of erythrocyte membranes and hemorheological disorders during oxidative stress caused by chronic physical overstrain in mice. Eksp. Klin. Farmakol. 74, 26-30 (in Russian)

Walker E. H., Pacold M. E., Perisic O., Stephens L., Hawkins P. T., Wymann M. P., Williams R. L. (2000): Structural determinants of phosphoinositide 3-kinase inhibition by wortmannin, LY294002, quercetin, myricetin, and staurosporine. Mol. Cell. 6, 909-919 http://dx.doi.org/10.1016/S1097-2765(05)00089-4

Xi Y. D., Yu H. L., Ding J., Ma W. W., Yuan L. H., Feng J. F., Xiao Y. X., Xiao R. (2012): Flavonoids protect cerebrovascular endothelial cells through Nrf2 and PI3K from $\beta$-amyloid peptide-induced oxidative damage. Curr. Neurovasc. Res. 9, 32-41 http://dx.doi.org/10.2174/156720212799297092

Zhang H., Forman H. J. (2008): Acrolein induces heme oxygenase1 through PKC-delta and PI3K in human bronchial epithelial cells. Am. J. Respir. Cell Mol. Biol. 38, 483-490 http://dx.doi.org/10.1165/rcmb.2007-0260OC 


\section{Reply from D. Margina and M. Ilie}

A great deal of scientific research has been focused lately on the role of food stuff in promoting health. There is an increased interest from both the consumers and researchers regarding the active role of food on the health state and on longevity. For example, the Mediterranean diet, flavonoid-rich, is considered to decrease the incidence of atherosclerotic based cardio-vascular disease and also to improve health in patients with hyperglycemia, obesity and insulin resistance - impairments with high incidence in modern societies. Quercetin and epigallocatechin gallate (EGCG) are among the most studied polyphenols in the frame of health-promoting food supplements (a search on PubMed with these terms returned as much as 1043 articles in 2011).

Several studies support the antioxidant potential of certain phenolic phytochemicals (including quercetin and EGCG); other disease-preventative characteristics have been pointed out in several studies, including in the Letter to editor by Dr. Chirumbolo (Procházková et al. 2011).

However, the research concerning the effects of flavonoids is mostly based on animal and cell line models, human studies having a lower extent; some of the results attributed to polyphenols come from extremely old, even mythological observational studies (as cited by Larson et al. 2012). It is difficult to presume, from a relatively small number of well designed and controlled human trials, how polyphenols improve the cardio-vascular function or the glucose homeostasis (Ostertag et al. 2010; Alexandru et al. 2012). Clinical studies which investigate the potential role of polyphenols in health and disease are difficult to design, as long-term studies are interfered by the treatment and the evolution of the disease. Another limitation comes from the fact that flavonoids are intensely metabolized in the gastro-intestinal tract and in the liver, and the initial polyphenol concentration remaining bio-available is under debate. Moreover, there are recent reports showing that differences between human microfloras are associated with qualitatively different patterns of metabolites in some flavonoids (Mladěnka et al. 2010). Also, there are interferences between different polyphenols at the level of metabolizing enzymes. For example, the methylation of green tea polyphenols in vivo may limit their direct effects. Quercetin acts as an inhibitor of catechol-O-methyltransferase (COMT) and is reported to increase the cellular absorption of EGCG and to decrease methylation rate; animal studies pointed out that quercetin induces a 2- to 3-fold increase of total and non-methylated EGCG in lung and kidney and an increasing trend in liver (Wang et al. 2012).

Another arguable point raises from the plasma polyphenol concentrations reached after normal dietary intake: some authors state that quercetin levels up to $10 \mu \mathrm{M}$ may be achieved when using an enriched diet (Tribolo et al. 2008; Margina et al. 2012a,b), but others consider $2 \mu \mathrm{M}$ as physiologically achievable and $10 \mu \mathrm{M}$ as supraphysiological concentrations (Winterbone et al. 2009; Jin et al. 2010).

Cell line studies have also limitation in translating the results to in vivo research. Among them, one can cite the fact that studies performed on cells have to take into account not only the parent polyphenol but also the effect of the main metabolites generated in humans. Comparative results showed for quercetin that the main metabolites (quercetin 3 '-sulfate, quercetin 3-glucuronide and 3'-methylquercetin 3-glucuronide) did not exhibit the anti-inflammatory effects that have been proved for quercetin on smooth muscle cells as well as on islet $\beta$-cells (Cho et al. 2012).

The mechanisms through which flavonoids modulate in vivo glucose homeostasis and influence the cardio-vascular function are not completed. Still, an analysis of 42 acute or short-term chronic clinical studies, that included 1297 participants, showed that flow-mediated dilatation and insulin resistance parameters was improved by chocolate or cocoa consumption, in association with a significant reductions in serum insulin (Hooper et al. 2012). Wedick et al. (2012) proved that a consumption of anthocyanin-rich foods, particularly blueberries and apples/pears was associated with a lower risk of type 2 diabetes in men and women. The antiobesity effect of EGCG, is supported by in vivo data, which have shown that EGCG decreased food uptake, lipid absorption, blood lipids and leptin levels, also stimulating energy expenditure, fat oxidation, high density lipoprotein levels, and fecal lipid excretion (Moon et al. 2007). Green tea catechins have a protective effect on weight gain (Brown et al. 2011).

As stated by Dr. Chirimbolo, polyphenols improve outcomes of inflammatory cardiovascular risk; for example, quercetin inhibits the in vitro proliferation of autoimmune T-cells and the expression of the proinflammatory cytokine TNF- $\alpha$ in human mononuclear cells (Sternberg et al. 2008). Experimental studies report an antihypertensive effect of flavonoids registered for hypertensive animal models but not for healthy ones, without a positive effects on elevated blood pressure in humans (Mladěnka et al. 2010).

Still, even if authors state that additional larger, longerduration and extensive trials are required to confirm the exact mechanism of cardiovascular benefits comong from polyphenols and their effects on glucose homeostasis and adipose tissue, incorporating plant foods that are rich in flavanols in the diet of healthy individuals could help to reduce cardio-vascular risk (Ostertag et al. 2010; Alexandru et al. 2012).

In conclusion, the influence of polyphenols on health and disease is far from being completely understood, even if their role in diabetes, obesity and cardiovascular disease has been documented. Research approaches to clarify their chemical- molecular mechanisms of action, role in normal 
and abnormal metabolic states as well as interaction with other agents (food, medicines, pollutants, etc.) are still needed, be they in silico, on fakes or other artificial models, in vitro and in vivo (in both animal and human). These could lead to a sound way of using polyphenol-rich diets in health and disease.

Denisa Margina and Mihaela Ilie

Carol Davila University of Medicine and Pharmacy, Faculty of Pharmacy, 6 Traian Vuia, sect 2, Bucharest, Romania

E-mail: m16ilie@yahoo.com mihaela.ilie@umf.ro

\section{References}

Alexandru N., Popov D., Georgescu A. (2012): Platelet dysfunction in vascular pathologies and how can it be treated. Thromb. Res. 129, 116-126 http://dx.doi.org/10.1016/j.thromres.2011.09.026

Brown A. L., Lane J., Holyoak C., Nicol B., Mayes A. E., Dadd T. (2011): Health effects of green tea catechins in overweight and obese men: A randomised controlled cross-over trial. Br. J. Nutr. 106, 1880-1889 http://dx.doi.org/10.1017/S0007114511002376

Cho J. M., Chang S. Y., Kim D. B., Needs P. W., Jo Y. H., Kim M. J. (2012). Effects of physiological quercetin metabolites on interleukin $1 \beta$-induced inducible NOS expression. J. Nutr. Biochem. (in press) http://dx.doi.org/10.1016/j.jnutbio.2011.08.007

Hooper L., Kay C., Abdelhamid A., Kroon P. A., Cohn J. S., Rimm E. B., Cassidy A. (2012): Effects of chocolate, cocoa, and flavan3-ols on cardiovascular health: A systematic review and metaanalysis of randomized trials. Am. J. Clin. Nutr. 95, 740-751 http://dx.doi.org/10.3945/ajcn.111.023457

Jin F., Nieman D. C., Shanely R. A., Knab A. M., Austin M. D., Sha W. (2010): The variable plasma quercetin response to 12-week quercetin supplementation in humans. Eur. J. Clin. Nutr. 64, 692-697 http://dx.doi.org/10.1038/ejen.2010.91

Larson A. J., Symons J. D., Jalili T. (2012): Therapeutic potential of quercetin to decrease blood pressure: review of efficacy and mechanisms. Adv. Nutr. 3, 39-46

Margina D., Ilie M., Gradinaru D. (2012a): Quercetin and epigallocatechin gallate induce in vitro a dose-dependent stiffening and hyperpolarizing effect on the cell membrane of human mononuclear blood cells. Int. J. Mol. Sci. 13, 4839-4859 http://dx.doi.org/10.3390/ijms13044839

Margina D., Ilie M., Manda G., Neagoe I., Mocanu M., Ionescu D., Gradinaru D., Ganea C. (2012b): Quercetin and epigallocatechin gallate effects on the cell membranes biophysical properties correlate with their antioxidant potential. Gen. Physiol. Biophys. 31, 47-55 http://dx.doi.org/10.4149/gpb_2012_005

Mladěnka P., Zatloukalová L., Filipský T., Hrdina R. (2010): Cardiovascular effects of flavonoids are not caused only by direct antioxidant activity. Free Radic. Biol. Med. 49, 963-975 http://dx.doi.org/10.1016/j.freeradbiomed.2010.06.010

Moon H. S., Lee H. G., Choi Y. J., Kimb T. G., Choa C. S. (2007): Proposed mechanisms of (-)-epigallocatechin-3-gallate for anti-obesity. Chem. Biol. Int. 167, 85-98 http://dx.doi.org/10.1016/j.cbi.2007.02.008

Ostertag L. M., O'Kennedy N., Kroon P. A., Duthie G. G., de Roos B. (2010): Impact of dietary polyphenols on human platelet function - a critical review of controlled dietary intervention studies. Mol. Nutr. Food Res. 54, 60-81 http://dx.doi.org/10.1002/mnfr.200900172

Procházková D., Boušová I., Wilhelmová N. (2011): Antioxidant and prooxidant properties of flavonoids. Fitoterapia 82, 513-523 http://dx.doi.org/10.1016/j.fitote.2011.01.018

Sternberg Z., Chadha K., Lieberman A., Hojnacki D., Drake A., Zamboni P., Rocco P., Grazioli E., Weinstock-Guttman B., Munschauer F. (2008): Quercetin and interferon-beta modulate immune response(s) in peripheral blood mononuclear cells isolated from multiple sclerosis patients. J. Neuroimmunol. 205, 142-147 http://dx.doi.org/10.1016/j.jneuroim.2008.09.008

Tribolo S., Lodi F., Connor C., Suri S., Wilson V. G., Taylor M. A., Needs P. A., Kroon P. A., Hughes D. A. (2008): Comparative effects of quercetin and its predominant human metabolites on adhesion molecule expression in activated human vascular endothelial cells. Atherosclerosis 197, 50-56 http://dx.doi.org/10.1016/j.atherosclerosis.2007.07.040

Wang P., Heber D., Henning S. M. (2012): Quercetin increased bioavailability and decreased methylation of green tea polyphenols in vitro and in vivo. Food Funct. (in press) http://dx.doi.org/10.1039/c2fo10254d

Wedick N. M., Pan A., Cassidy A., Rimm E. B., Sampson L., Rosner B. (2012): Dietary flavonoid intakes and risk of type 2 diabetes in US men and women. Am. J. Clin. Nutr. 95, 925-933 http://dx.doi.org/10.3945/ajcn.111.028894

Winterbone M. S., Tribolo S. Needs P. W., Kroon P. A., Hughes D. A. (2009): Physiologically relevant metabolites of quercetin have no effect on adhesion molecule or chemokine expression in human vascular smooth muscle cells. Atherosclerosis 202, $431-438$ http://dx.doi.org/10.1016/j.atherosclerosis.2008.04.040 